\title{
Mucosectomía endoscópica para cáncer gástrico temprano: una alternativa vigente
}

\section{Endoscopic mucosal resection for early gastric cancer: A current alternative}

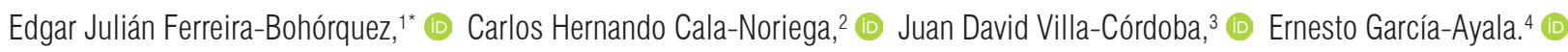

\author{
Gacceso abierto \\ Citación: \\ Ferreira-Bohórquez EJ, Cala-Noriega CH, Villa- \\ Córdoba JD, García-Ayala E. Mucosectomía \\ endoscópica para cáncer gástrico temprano: \\ una alternativa vigente. Rev Colomb \\ Gastroenterol. 2021:36(1):126-129. https://doi. \\ org/10.22516/25007440.492 \\ Gastroenterólogo clínico-quirúrgico, Hospital \\ Regional Manuela Beltrán del Socorro. Socorro, \\ Santander, Colombia. \\ 2 Médico de urgencias, Hospital Regional \\ Manuela Beltrán. Socorro, Santander, Colombia. \\ ${ }^{3}$ Médico interno, Universidad Nacional de \\ Colombia. Bogotá, Colombia. \\ ${ }^{4}$ Médico patólogo y docente de la Universidad \\ Industrial de Santander (UIS). Bucaramanga, \\ Santander, Colombia. \\ *Correspondencia: \\ Edgar Julián Ferreira-Bohórquez. \\ ejulferbo@yahoo.com \\ Fecha recibido: $\quad 17 / 12 / 19$ \\ Fecha aceptado: $17 / 06 / 20$
}

\begin{abstract}
Resumen
La mucosectomía endoscópica ha probado ser una alternativa efectiva para el tratamiento del cáncer gástrico temprano; sin embargo, se requiere de un seguimiento estricto para asegurar una conducta de manejo adecuada en caso de recurrencia. Se presenta el caso de un paciente de 54 años, con hallazgo incidental de un carcinoma gástrico temprano, a quien se le realizó mucosectomía endoscópica, con un reporte final de histopatología compatible con adenocarcinoma gástrico temprano y márgenes de resección negativos, con un seguimiento endoscópico e histopatológico a 2 meses negativos para malignidad.
\end{abstract}

\section{Palabras clave}

Cáncer gástrico, resección endoscópica de la mucosa.

\begin{abstract}
Endoscopic mucosal resection has proven to be an effective alternative for the treatment of early gastric cancer. However, strict follow-up is necessary to ensure adequate management in the event of recurrence. This is the case of a 54-year-old patient with an incidental finding of early gastric carcinoma. He underwent endoscopic mucosal resection, with a final histopathology report compatible with early gastric adenocarcinoma and negative resection margins. Endoscopic and histopathological follow-up at 2 months showed negative results for malignancy.
\end{abstract}

Keywords

Gastric cancer, Endoscopic mucosal resection.

\section{INTRODUCCIÓN}

El cáncer gástrico temprano se define como aquel cáncer cuya invasión se limita a la capa submucosa del estómago al examen microscópico, independiente del compromiso de ganglios linfáticos. Esta definición fue propuesta por la Sociedad Japonesa de Endoscopia Digestiva en 1971 (1). Desde hace cerca de 3 décadas, se conoce que la supervivencia a 5 años es del $100 \%$ en pacientes con lesiones en la mucosa y $90 \%$ en los pacientes con invasión submucosa. También se ha descrito supervivencia del $99 \%$ en pacientes con clasificación $\mathrm{N}_{0}$ y de $73 \%$ con clasificación $\mathrm{N}_{1}$. Los datos de supervivencia a 10 años son idénticos en pacientes sometidos a manejo quirúrgico o endoscópico para el cáncer gástrico temprano $(2,3)$. 
En 2018 el cáncer gástrico representó más de un millón de nuevos casos a nivel mundial y un estimado de muertes que asciende a 783000 , por lo que esta entidad se ubicó como la tercera causa de muerte por cáncer (4).

Una de las clasificaciones más ampliamente utilizadas es la de París de 2002, en la que cada tipo es predictivo del grado de invasión en la submucosa, que se correlaciona con el riesgo de metástasis ganglionares en las lesiones gástricas. Las lesiones clasificadas como París 0-I, 0-IIc y 0-III se asocian con un mayor riesgo de invasión submucosa ( $57 \%$, $37 \%$ y $40 \%$, respectivamente) en comparación con las lesiones 0-IIa y 0-IIb (29\% y $20 \%$, respectivamente) (Tabla 1) (5).

Tabla 1. Clasificación de París

\begin{tabular}{|c|c|c|}
\hline $\begin{array}{l}\text { Características } \\
\text { endoscópicas }\end{array}$ & Tipo & Descripción \\
\hline Lesiones polipoides & $\begin{array}{l}0-1 p \\
0-I s\end{array}$ & $\begin{array}{l}\text { Pólipos pedunculados } \\
\text { Pólipos sésiles }\end{array}$ \\
\hline $\begin{array}{l}\text { Lesiones no } \\
\text { polipoides }\end{array}$ & $\begin{array}{l}0-\| l a \\
0-\| l b \\
0-\| l c\end{array}$ & $\begin{array}{l}\text { Superficial elevada } \\
\text { Completamente plana } \\
\text { Superficial, deprimida sin ulceración }\end{array}$ \\
\hline Lesiones excavadas & $0-\mid I I$ & Excavadas y ulceradas \\
\hline
\end{tabular}

En Colombia se recomienda la realización de endoscopia digestiva alta con luz blanca, en todos los pacientes con dispepsia no investigada, si tienen síntomas y signos de alarma; y también se utiliza en asociación la cromoendoscopia con colorantes tipo índigo carmín para el tamizaje y detección de lesiones premalignas y tumores gástricos tempranos. También se utiliza el mapeo con protocolo de Sydney y el estudio histológico con protocolo OLGA (operative link for gastritis assessment) (6).

\section{CASO CLÍNICO}

Se presenta el caso de un hombre de 54 años procedente del área rural de Socorro, Santander, a quien se le realizó una endoscopia digestiva alta para la evaluación por un posible cuerpo extraño esofágico, y se encontró como hallazgo incidental la presencia de una lesión de $8 \mathrm{~mm}$ en el antro hacia la curva mayor de $8 \mathrm{~mm}$ de diámetro, altamente compatible con una neoplasia gástrica temprana 0-Is (Figura 1). Las biopsias fueron compatibles con adenocarcinoma bien diferenciado. Con dichos hallazgos consultó al Hospital Regional Manuela Beltrán, donde se complementaron los estudios con una ultrasonografía endoscópica gástrica, que evidenció el compromiso de las capas ultrasonográficas I y II (hasta la muscular de la mucosa) (Figura 2); por tanto, se clasificó como uTluN0, compatible con un cáncer gástrico temprano. Como estudios adicionales, una tomografía axial computarizada (TAC) de abdomen contrastada descartó la presencia de metástasis a distancia o compromiso ganglionar. Se programó para realizarle una mucosectomía endoscópica gástrica.

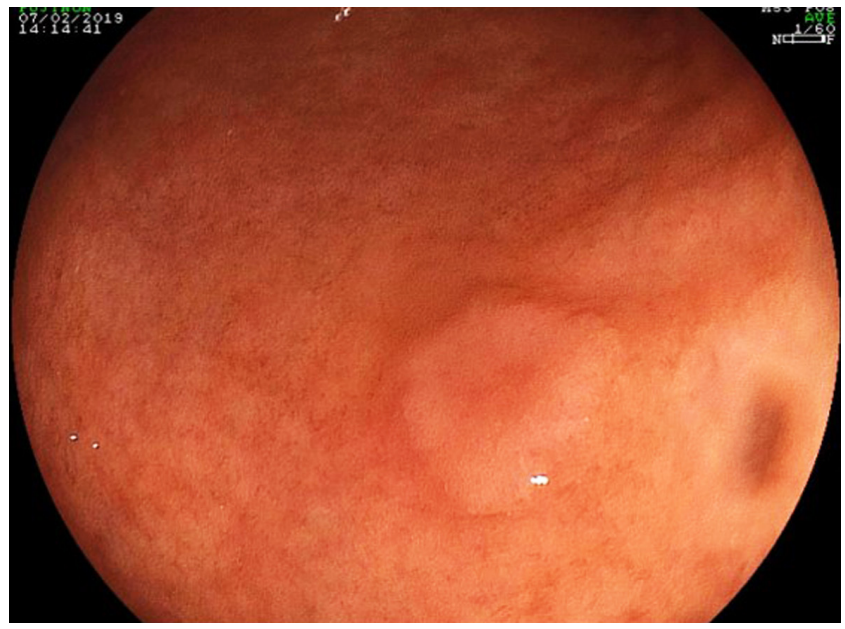

Figura 1. Lesión 0-Is encontrada como hallazgo incidental en la endoscopia de vías digestivas altas.

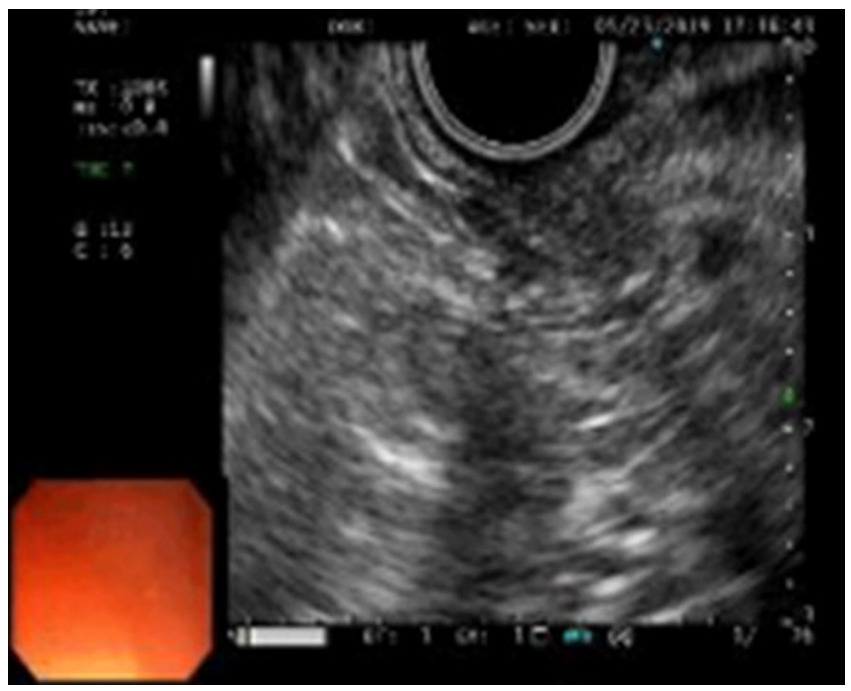

Figura 2. Ultrasonografía endoscópica, lesión que llega hasta la segunda capa.

\section{Descripción del procedimiento y seguimiento}

Se utilizó una torre de endoscopia estándar, con esofagogastroduodenoscopio de un solo canal de trabajo. Se utilizó una aguja de esclerosis con solución de adrenalina 1:20 000 para infiltrar la lesión en su base y se practicó una resección endoscópica con un asa poligonal, con la que se logró recuperar una sola pieza completa, de aproximadamente $12 \mathrm{~mm}$ de dimensión mayor (Figuras 3 y 4 ). 


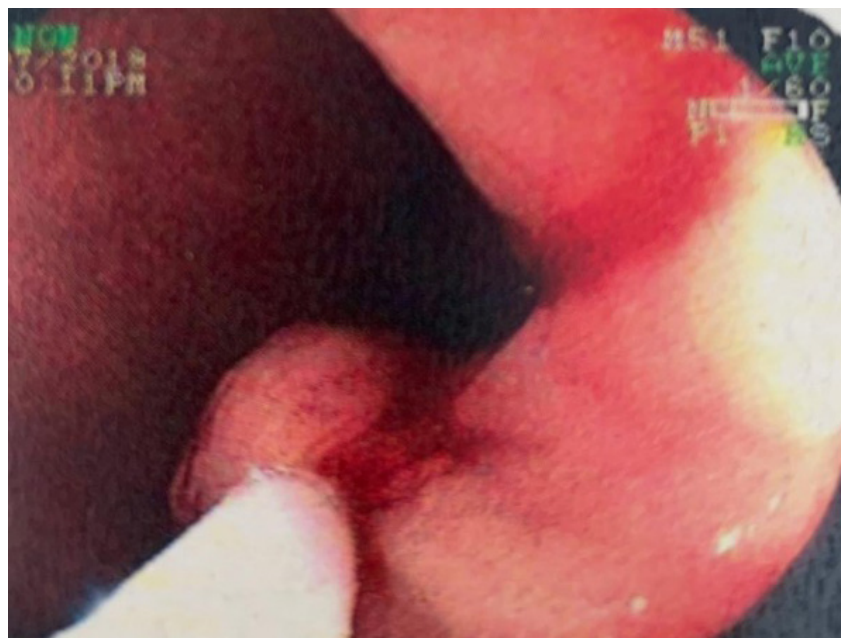

Figura 3. Resección durante la mucosectomía.

Se envió la muestra para el estudio histopatológico. El resultado de la patología fue reportado de la siguiente manera: "Resección endoscópica de la lesión (pared gástrica) con adenocarcinoma gástrico temprano (precoz, superficial, bien diferenciado) de variedad intestinal que compromete hasta la muscular de la mucosa sin traspasarla. Líneas de sección laterales y borde medial alejados y libres de lesión" (Figuras 5 y 6 ).

El paciente tuvo una evolución clínica satisfactoria y tuvo egreso hospitalario un día después del procedimiento. Se realizó una endoscopia digestiva alta de control a los 2 meses de la resección endoscópica en la que se hallaron

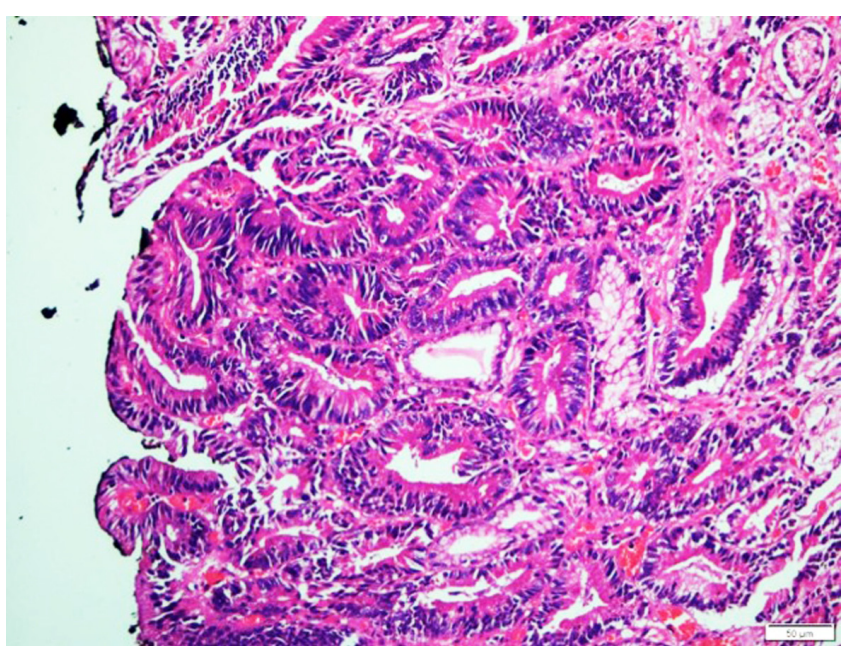

Figura 5. 10x hematoxilina-eosina. Mucosa gástrica con reemplazo de la arquitectura normal por presencia de lesión tumoral maligna conformada por elementos glandulares y tubulares, revestidos por epitelio columnar alto focalmente pseudoestratificado con moderado pleomorfismo e hipercromatismo nuclear.

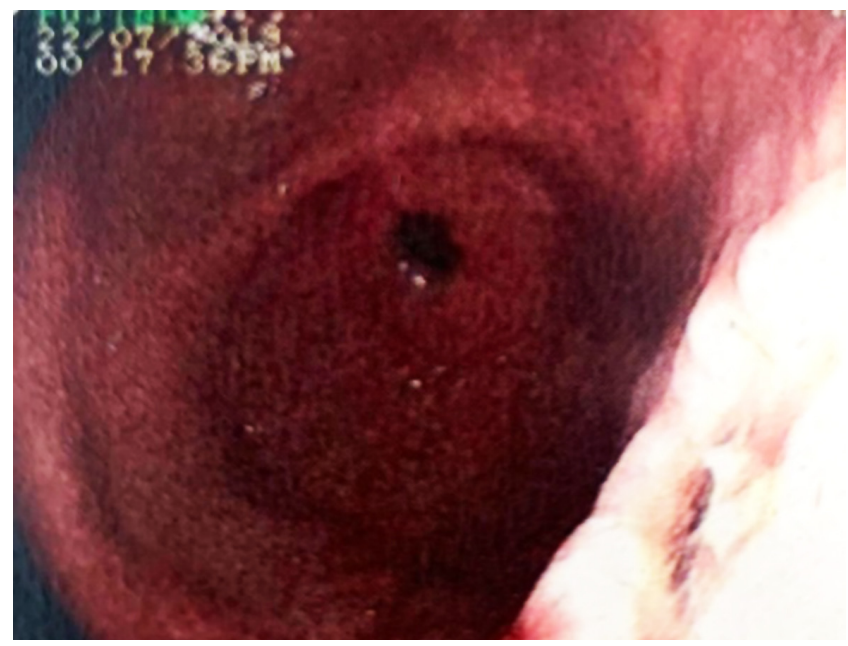

Figura 4. Lecho de la lesión.

cambios cicatriciales sobre el lecho de resección y se tomaron biopsias múltiples que fueron reportadas sin evidencia de malignidad. El paciente se encuentra asintomático y en espera de un nuevo seguimiento endoscópico.

\section{DISCUSIÓN}

La mucosectomía endoscópica o resección endoscópica de la mucosa se considera una alternativa segura para el tratamiento de lesiones superficiales limitadas a la mucosa y cuenta con una tasa de resección completa mayor del $90 \%$

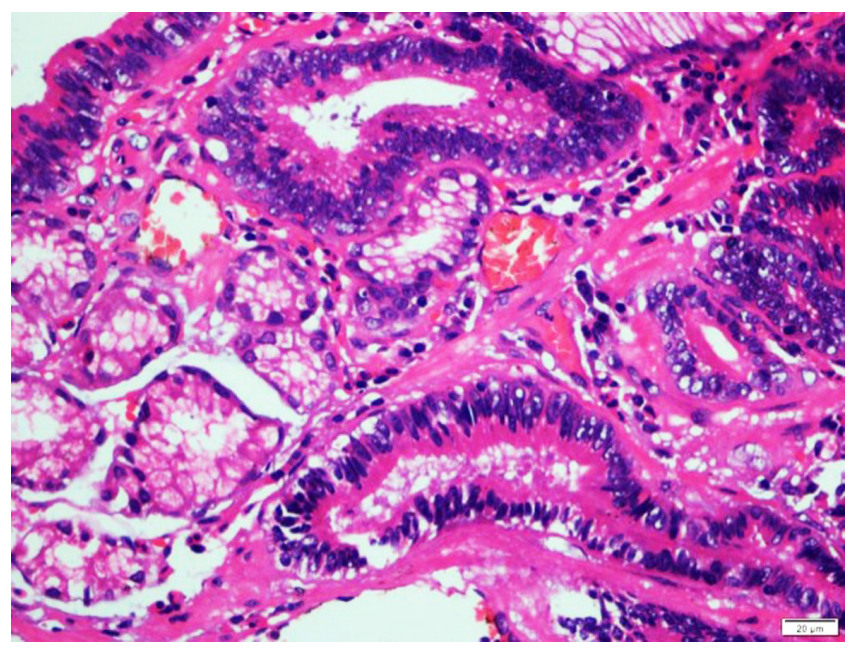

Figura 6. 40x hematoxilina-eosina. Se observan elementos glandulares tubulares en cercanía de fibra muscular lisa de la muscular de la mucosa. Adyacente se encuentra componente glandular normal. 
de los casos. Existe también la disección endoscópica de la submucosa, reservada para lesiones mayores de $20 \mathrm{~mm}$, y más profundas tipo IIc y III, el cual es un procedimiento más complejo, pero que cuenta con tasas de éxito superiores al $95 \%$. Las complicaciones del tratamiento endoscópico son muy raras; el sangrado y la perforación son las más frecuentes (hasta $5 \%$ ), y con menor frecuencia son el enfisema subcutáneo y neumomediastino (7-9).

Se recomienda el seguimiento de los pacientes tratados por cáncer gástrico en donde pueda realizarse una endoscopia cada 3 a 6 meses por 1 a 2 años, luego cada 6 a 12 meses por 3 a 5 años $y$, finalmente, cada año (8).

\section{CONCLUSIONES}

El avance en las técnicas de diagnóstico y terapia endoscópica, incluidos el ultrasonido endoscópico y diferentes técnicas de resección endoscópica, han permitido que actualmente el cáncer gástrico temprano pueda ser tratado con medidas poco invasivas y tasas de éxito altas. No obstante, se requiere un seguimiento endoscópico periódico estricto o para supervisar recurrencias y reintroducir un tratamiento oportuno adecuado.

\section{REFERENCIAS}

1. Murakami T. Pathomorphological diagnosis: definition and gross classification of early gastric cancer. Gann Monogr Cancer Res. 1971;11:53-5.

2. Inoue K, Tobe T, Kan N, Nio Y, Sakai M, Takeuchi E, Sugiyama T. Problems in the definition and treatment of early gastric cancer. Br J Surg. 1991;78(7):818-21. https// doi.org/10.1002/bjs.1800780717

3. Han S, Hsu A, Wassef WY. An update in the endoscopic management of gastric cancer. Curr Opin Gastroenterol. 2016;32(6):492-500. https//doi.org/10.1097/ MOG.0000000000000318

4. Bray F, Ferlay J, Soerjomataram I, Siegel RL, Torre LA, Jemal A. Global cancer statistics 2018: GLOBOCAN estimates of incidence and mortality worldwide for 36 cancers in 185 countries. CA Cancer J Clin. 2018;68(6):394-424. https//doi.org/10.3322/caac.21492

5. The Paris endoscopic classification of superficial neoplastic lesions: esophagus, stomach, and colon: November 30 to December 1, 2002. Gastrointest Endosc.
2003;58(6 Suppl):S3-43. https//doi.org/10.1016/ s0016-5107(03)02159-x

6. Gómez MA, Riveros JH, Ruiz O, Concha A, Ángel DM, Torres M, Pardo R, Otero W, Sabbagh L. Guía de práctica clínica para la prevención, diagnóstico y tratamiento del cáncer gástrico temprano-2015. Rev Col Gastroenterol. 2015;30(Supl 1):34-42.

7. Espinel J, Pinedo E, Ojeda V, Del Rio MG. Treatment modalities for early gastric cancer. World J Gastrointest Endosc. 2015;7(12):1062-9. https//doi.org/10.4253/ wjge.v7.i12.1062

8. Gómez M, Otero W, Arbeláz V. Tratamiento endoscópico de cáncer gástrico temprano en Colombia con seguimiento a cinco años. Rev Col Gastroenterol. 2009;24(4):347-52.

9. Ono H, Kondo H, Gotoda T, Shirao K, Yamaguchi H, Saito D, Hosokawa K, Shimoda T, Yoshida S. Endoscopic mucosal resection for treatment of early gastric cancer. Gut. 2001;48(2):225-9. https//doi.org/10.1136/gut.48.2.225 